\title{
HARECES Observations of Magnetic Linear Dichroism in a Nickel Ferromagnet
}

\author{
Nestor J.Zaluzec ${ }^{1,2}$, Yasuo Ito ${ }^{2}$, Russell E.Cook ${ }^{1}$, Dean J.Miller ${ }^{1}$ \\ ${ }^{1}$ Electron Microscopy Center, Materials Science Div. , Argonne National Laboratory, Argonne IL \\ ${ }^{2}$ Dept. of Physics, Northern Illinois University, DeKalb IL.
}

With the development of the spin-based electronics and nanoscale manipulation of structure, tremendous efforts have been focused on fabrication and characterization of nanoscale devices. Since the spin transport occurs through the bulk of multi-layered nanostructures, techniques that are directly sensitive to the magnetic anisotropy, within a specimen and at its internal interfaces, are of great interest, particularly those which operate at high spatial resolution.

Magnetic Linear Dichroism (MLD) is an often-used method to study magnetism, which is facilitated, by studying changes in X-ray Absorption Near Edge Structure (XANES) measured at Synchrotron radiation sources. In an electron scattering experiment, a similar spectroscopy namely: Electron Loss Near Edges Structure (ELNES) can be also employed to study MLD. In this case, we make use of the fact that the perturbing electric field (of an incident electron probe) is longitudinally polarized in the direction parallel to the momentum transfer and thus can be used to probe the electronic and magnetic structure of a material (fig. 1). The interest in exploring this measurement using an electron microscope arises due to the added advantage that in these instruments the spatial resolution is far superior to that of Synchrotron sources. MLD in an electron microscope is realized at ANL by using the high angular resolution electron channeling electron spectroscopy (HARECES) facilities in the ANL EM Center[1]. In our initial work we have verified the ability of our methods to measure MLD, by studying the changes in core loss spectra taken from material in different states of a ferromagnetic Nickel specimen. Previous work using this concept has been performed only on anti-ferromagnets.[2-3] As in XANES, we determine MLD, by measuring the intensity of $\mathrm{p} \rightarrow \mathrm{d}$ transitions in L-shell spectra of transition metals (fig. 2).

Our angle-resolved EELS measurements on electropolished Nickel along were performed this work using a FEI Tecnai F-20 electron microscope operating at $200 \mathrm{kV}$, by employing a small collection aperture $\beta$ of $<0.3 \mathrm{mrad}$ together with a highly coherent and nearly parallel probe $(\alpha<0.05 \mathrm{mrad})$. The diameter of this probe used during these measurements was typically less than 0.5 micron, which was used to define the region of interest on a nearly uniformly thick single crystal region of an oriented $\{001\}$ grain. Prior to analysis the sample was cleaned for 15 minutes in Argon using a SouthBay Technology PC2000 plasma cleaner to mitigate the deleterious effects of hydrocarbon contamination, which was not detectable during the course of our measurements.

Figure 3 presents our results demonstrating the ability to measure MLD in ferromagnetic Nickel by observing the changes in the Ni L shell edge intensity. Here we measure the L shell profiles above and below the Curie temperature of Nickel along the forward scattering direction. Below the Curie temperature (blue) the sample is magnetic and the domains lie in plane due to shape anisotropy of the thin Ni film. Above the Curie temp, the domains become randomized and the fraction lying out of plane increase resulting in a small but measurable change in the L shell $\mathrm{p}->\mathrm{d}$ transition. In figure 4 we present additional confirming evidence of the magnetic state of the material by plotting the relative normalized $\mathrm{Ni} \mathrm{L}$ shell intensity as a function of in plane orientation. Here above the 
transition temperature the intensity distribution more isotropic, than corresponding measurements below, which can be attributed to the formation of in-plane magnetic domains.

In continuation of this work, we will be determining the optimum conditions for measuring MLD using HARECES in order that we can establish this technique as a routine method for high spatial resolution magnetic measurements.

\section{References}

[1] N. J. Zaluzec, etal, Microscopy and Microanalysis, 11, S2, 718. (2005)

[2] J. Yuan and N.K. Menon, J. Appl. Phys. 81 (8), 15 (1997)

[3] P. A. van Aken, S. Lauterbach, Phys Chem. Minerals 30: 469 (2003)

[4] This work was supported in part by the U.S. DOE under BES-MS W-31-109-Eng-38 at ANL and by the InSET Program at NIU.

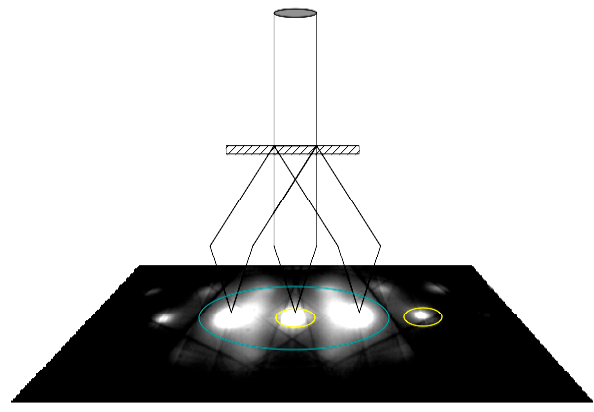

Figure 1. Traditional EELS measurements are performed using large apertures and poor angular resolution $(\sim 2-10 \mathrm{mR})$. In HARECES we employ computationally mediated control of the electron microscope which allows spectroscopic measurements at angular resolutions as small as $0.05 \mathrm{mR}$.

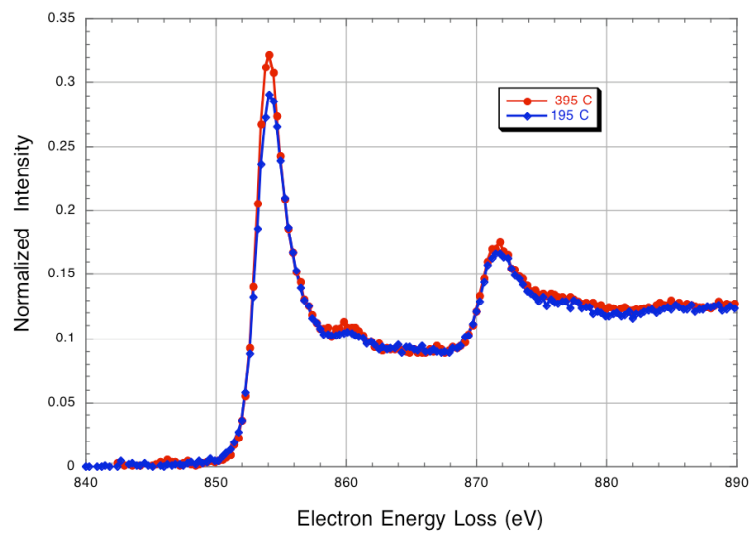

Figure 3. Nickel L-shell spectra recorded above (red-upper) and below (blue-lower) the Curie temperature to verify that HARECES based MLD is sensitive to magnetic state. Spectra were normalized at the pre L2 edge plateau, the pre L shell edge background was modeled and subtracted using the conventional model of $\mathrm{Bgnd}=\mathrm{AE}^{-\mathrm{r}}$

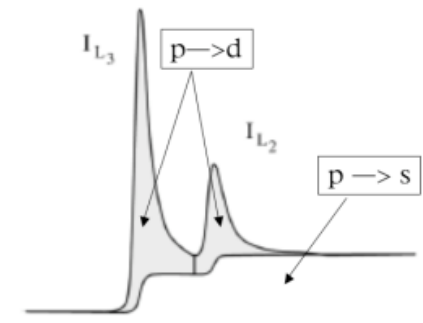

Figure 2. L-shell spectra are made up of 2 components $\mathrm{p}->\mathrm{s}$ and $\mathrm{p}->\mathrm{d}$ transitions. The $\mathrm{p}->\mathrm{d}$ components are those which are sensitive to the magnetic state of the material. 